\title{
On recent results in the modelling of neoclassical-tearing-mode stabilization via electron cyclotron current drive and their impact on the design of the upper EC launcher for ITER
}

\author{
E. Poli ${ }^{1}$, C. Angioni ${ }^{1}$, F. J. Casson ${ }^{1, *}$, D. Farina ${ }^{2}$, L. Figini ${ }^{2}$, T. P. Goodman ${ }^{3}$, \\ O. Maj ${ }^{1}$, O. Sauter ${ }^{3}$, H. Weber ${ }^{1}$, H.Zohm ${ }^{1}$, G. Saibene ${ }^{4}$, M. A. Henderson ${ }^{5}$ \\ ${ }^{1}$ Max-Planck-Institut für Plasmaphysik, Garching, Germany \\ ${ }^{2}$ Istituto di Fisica del Plasma CNR, Milano, Italy \\ ${ }^{3}$ Centre de Recherches en Physique des Plasmas, CRPP-EPFL, Lausanne, \\ Switzerland \\ ${ }^{4}$ Fusion for Energy, Barcelona, Spain \\ ${ }^{5}$ ITER Organization, Saint-Paul-Lez-Durance, France
}

\begin{abstract}
Electron cyclotron wave beams injected from a launcher placed in the upper part of the vessel will be used in ITER to control MHD instabilities, in particular neoclassical tearing modes (NTMs). Simplified NTM stabilization criteria have been used in the past to guide the optimization of the launcher. Their derivation is reviewed in this paper and their range of applicability clarified. Moreover, possible effects leading to a deterioration of the predicted performance are discussed. Particularly critical in this context is the broadening of the EC deposition profiles. It is argued that the most detrimental effect for ITER is likely to be the scattering of the EC beams from density fluctuations due to plasma turbulence, resulting in a beam broadening by about a factor of two. The combined impact of these effects with that of beam misalignment (with respect to the targeted surface) is investigated by solving the Rutherford equation in a form that retains the most relevant terms. The perspectives for NTM stabilization in the $Q=10$ ITER scenario are discussed.
\end{abstract}

\section{Introduction}

The electron-cyclotron (EC) heating and current drive (CD) system envisaged for ITER consists of two launchers, an equatorial launcher (EL) designed primarily for central heating, current drive and profile tailoring, and an upper launcher (UL) whose main goal is the control of magnetohydrodynamical instabilities, in particular the neoclassical tearing mode (NTM) and the sawtooth instability [1]. The power is provided by 24 gyrotrons delivering $1 \mathrm{MW}$ each (with optional upgrade to $2 \mathrm{MW}$ in a later phase) at a frequency of $170 \mathrm{GHz}$. The nominal power reaching the plasma on account of transmission losses is 20 MW, to be divided between the two launchers. This paper focuses on the criteria guiding the design of the UL, which is being developed in the frame of a European collaboration [2] and has reached recently the preliminary design level, i. e. the last major step before design finalization [3]. The UL comprises four ports, each housing eight beam lines, arrayed in an upper and a lower row of four waveguides each. Each row is provided with independent steering mechanisms, referred to as the Upper Steering Mirror (USM) and Lower Steering Mirror (LSM). The power which can be launched from each row amounts hence to $13.3 \mathrm{MW}$.

To take full advantage of the high localization of the interaction between EC beams and the plasma, which is particularly important for the applications envisaged for the UL, it is essential that the beams retain good focusing properties over the whole spatial domain where their use can be expected for different plasma scenarios. In the early development stage of the launcher, it was found that a design based on remote steering [4], although

*Present address: CCFE, Culham Science Centre, Abingdon, UK 
attractive because movable parts would have been placed in a secondary vacuum away from the plasma, was unable to deliver sufficiently focused beams over the whole steering range. The adoption of a front steering concept [5] has allowed a larger flexibility in the design of the beams, which in turn has led to an optimization of the distribution of the tasks between EL and UL [6].

The main requirement to be fulfilled by the UL is that the beams launched from it into the ITER plasma be able to suppress NTMs. During the design phase, several iterations are necessary to ensure that the required physical performance is achieved without violating the technical constraints posed by the ITER environment. Simple formulas, that allow one to check whether the physics goals are achieved, are extremely useful, also because their inspection usually provides a direct hint to the quantities that should be optimized by the system. A first criterion to determine the EC power needed to achieve this goal, based on the generalized Rutherford equation [7, 8], was put forward by Zohm et al. [9]. In the derivation of this criterion (cf. Sec. 2 below) it was assumed that the EC deposition profile would be wide compared to the marginal magnetic-island size (i. e. the size at which the island self-stabilizes). This situation was usually found in beam tracing calculations employing remote-steering beam parameters. A further reason for assuming wide EC profiles as compared to the marginal island size was its scaling with the normalized ion gyroradius $\rho_{*}$ [10], which implies small marginal island widths for ITER. The criterion derived by Zohm et al. states that the driven current density should exceed by $20 \%$ the (unperturbed) bootstrap current density at the rational surface where the NTM develops. In this case of wide deposition profiles, the EC power should be modulated such as to heat the O-point region, while no power is delivered when the X-point is in front of the antenna. The optimization of the driven current density has led to the determination of an optimum toroidal injection angle $\beta=20^{\circ}$. This is a parameter to be fixed in the design of the UL (beam steering is achieved through variation of the poloidal injection angle $\alpha$ ). On the other hand, ray/beam tracing calculations indicate that the high focusing capabilities of a launcher based on front steering could allow EC deposition profiles below the marginal island width. In this respect, it was noted by Sauter et al. [11] that, once the beams deposit their power well inside the island, further focusing does not result in significantly higher stabilization efficiency (making the process in fact more sensitive to a possible misalignment between beam and island). For this reason, it was proposed to optimize in this case the total driven current, adding the constraint that the EC deposition width should not exceed $5 \mathrm{~cm}$. For such narrow deposition profiles, continuous wave $(\mathrm{cw})$ injection has a stabilization efficiency only sightly lower than that of modulated injection. These results were confirmed later in Ref. [12]. Consequently, it was suggested that more efficient NTM stabilization would be achieved by a small increase of the toroidal injection angle, in particular for the LSM, since this would increase the total driven current without losing localization.

At the same time, various physical processes that could spoil the narrowness of the EC power deposition predicted by standard ray/beam tracing codes have been studied, like for instance aberration [13, 14, 15], radial transport of EC-heated electrons [16], beam scattering from density fluctuations $[17,18]$ (other effects are also discussed later in this paper). A quantitative prediction of these effects in ITER is indispensable in the frame of the UL optimization outlined above: while a profile broadening in the $10 \%$ range would not affect significantly the ratio between deposition width and marginal island size, this would be the case for a $100 \%$ broadening. This assessment has several implications. First of all, it should be determined whether the power required for complete NTM suppression remains within the capabilities of the system. Furthermore, it affects the decision as to whether modulation capabilities should be retained (which opens also the question of the detailed dynamical model of the ECCD process, as the island rotation frequency in the laboratory frame and the collision frequency on which ECCD establishes could be comparable $[19,20])$. Finally, the profile width also affects the steering accuracy required to compensate possible offsets of the deposition profile with respect to the island. 
These issues are addressed in this paper, which reports on part of the theory work performed in support of the UL design activities in the frame of the European ECHUL consortium [21]. This activity benefits from the progress of the entire community on this subject, so that a consistent part of this article is devoted to a review of results obtained in the last years. At the same time, some very recent progress achieved in this field, partly still in the course of publication, is presented here for the first time in its context. Moreover, the insight gained through this analysis is exploited in the final part of the paper to derive some new results concerning the optimization of the UL parameters and the expected performance for NTM stabilization. The paper is structured as follows. A common derivation of the NTM stabilization criteria mentioned above is presented, their validity range elucidated and their accuracy discussed in Sec. 2. In Sec. 3, the role of different effects that can lead to a broadening of the EC profiles is addressed and a quantitative estimate of the change of the EC profile width is given. These results are used as boundary conditions for an evaluation of the impact of profile broadening and misalignment on the power required for NTM suppression (or at least reduction of the island size below a given threshold) in Sec. 4. Summary and conclusions are drawn in Sec. 5 .

\section{Derivation of the stabilization criteria}

In the derivation of simple stabilization criteria for the neoclassical tearing mode in the presence of EC heating and current drive, the first step is to simplify the generalized Rutherford equation $[7,8]$, which describes the temporal evolution of the island width, such that only the most relevant terms describing the process are retained. A conclusion common to several studies about EC stabilization of NTMs in ITER $[9,11,12]$ is that these terms are those involving the classical stability parameter $\Delta^{\prime}[22]$, the bootstrap current destabilization $[23,24]$ (possibly reduced on account of geodesic curvature effects $[25,26])$ and the effect of the helical component of the EC current on the island evolution [27]. Small-island effects are usually included as a transport threshold on the bootstrap term [28] or through the polarization-current term [29].

As mentioned in Sec. 1, the first simple quantitative criterion for a complete stabilization of NTMs used to guide the design and assess the performance of the ITER upper launcher was formulated as [9]

$$
\eta_{N T M} \equiv \frac{j_{\mathrm{CD}}}{j_{\mathrm{bs}}}>1.2,
$$

where $j_{\mathrm{CD}}$ and $j_{\mathrm{bs}}$ are the peak ECCD-driven current density and the bootstrap current density at the surface of interest, respectively. Successively, it was proposed to employ a different criterion, namely $[11,12]$

$$
\eta_{N T M} w_{\mathrm{CD}}>5 \mathrm{~cm}, \quad w_{\mathrm{CD}}<5 \mathrm{~cm},
$$

where $w_{\mathrm{CD}}$ is the full width (at $1 / e$-level) of the ECCD profile. In this paper, we will refer to Eq. (1) as Zohm's criterion and to Eq. (2) as Sauter's criterion. Both can be derived from a simplified form of the Rutherford equation under different assumptions on the width of the ECCD profile in the sense specified below. This derivation, that follows closely that of Ref. [9], being based on elementary analytic manipulations of a simplified Rutherford equation, complements the analyses reported in Refs. [11, 12], which are mainly based on a numerical solution of a more complete form of the Rutherford equation. The approach described below allows a straightforward clarification of the validity range of both criteria and their formulation in dimensionless form (a dimensionless analysis has been used previously also in Ref. [12]). The discussion presented in this section also aims to verify that a simple form of the Rutherford equation, see Eq.(10) below, reproduces correctly the main trends observed with a more complete modelling and can hence be employed for the 
calculations presented in Sec. 4, which include the impact of the broadening of the ECCD profile and of the possible misalignment between the resonant surface and the position at which the maximum EC current is driven.

The condition for a stable island size $\mathrm{d} w / \mathrm{d} t=0$ ( $w$ is the island full width) can be cast in the form

$$
0=-1+\frac{w_{\mathrm{sat}}}{w}-5.05 \frac{w_{\mathrm{CD}} w_{\mathrm{sat}}}{w^{2}} \frac{j_{\mathrm{CD}}}{j_{\mathrm{bs}}} \eta_{\mathrm{CD}},
$$

where the saturated island width $w_{\text {sat }}$ expresses the balance between the neoclassical (bootstrap) drive and the stabilization due to the equilibrium current profile in the absence of ECCD, and the CD stabilization efficiency $\eta_{\mathrm{CD}}$, defined as $[27,19]$

$$
\eta_{\mathrm{CD}}=\frac{\int_{-1}^{\infty} \mathrm{d} \Omega\langle 1\rangle_{\Omega}^{-1}\langle\cos \xi\rangle_{\Omega}\left\langle J_{\mathrm{CD}}\right\rangle_{\Omega}}{\int_{-1}^{\infty} \mathrm{d} \Omega\left\langle J_{\mathrm{CD}}\right\rangle_{\Omega}},
$$

weights the component of the driven current with the correct helicity for mode stabilization. In the previous equation, the flux surface average operator

$$
\langle\ldots\rangle_{\Omega}=\oint \frac{\mathrm{d} \xi}{2 \pi} \frac{\ldots}{\sqrt{\Omega+\cos \xi}}
$$

has been introduced, where the helical flux label $\Omega$ is defined as $\Omega=8 x^{2} / w^{2}-\cos \xi$ and $\xi$ is the helical angle ( $x$ is the distance from the rational surface). Eq. (3) is kept in its simplest form, neglecting stabilizing effects at small island width and possible weighting coefficients in front of the various terms. Requiring that no roots of Eq. (3) exist (unconditional stability), i. e. that its discriminant is negative, leads to the following criterion for NTM stabilization:

$$
\frac{w_{\mathrm{CD}}}{w_{\mathrm{sat}}} \frac{j_{\mathrm{CD}}}{j_{\mathrm{bs}}} \eta_{\mathrm{CD}}>\frac{1}{20} .
$$

It is useful to distinguish between the case in which the ECCD profile is broader or narrower than the typical island width $w_{\text {marg }}$ at which stabilization occurs. In the former limit, $w_{\mathrm{CD}}>w_{\text {marg }}$, the $\mathrm{CD}$ stabilization efficiency can be approximated as $\eta_{\mathrm{CD}} \simeq$ $0.15 w / w_{\mathrm{CD}}$ for modulated injection in the O-point (50\% duty cycle) and $\eta_{\mathrm{CD}} \simeq w^{2} / 8 w_{\mathrm{CD}}^{2}$ for continuous injection [9]. To express the stabilization condition it is more practical to exploit the fact that in ITER it can be assumed that $w_{\text {marg }}$ is much smaller than $w_{\text {sat }}$, so that the first term on the right-hand side of Eq. (3) can be dropped near marginal stability. In case of modulated injection, Eq. (3) leads then directly to the result that stabilization is achieved if

$$
\eta_{N T M}>\frac{4}{3} .
$$

This condition is very similar to Zohm's criterion given above (1), which includes a reduction of the neoclassical drive due to geodesic-curvature effects [25, 26]. For continuous injection, the previous criterion should be replaced by

$$
\frac{j_{\mathrm{CD}}}{j_{\mathrm{bs}}}>\frac{8}{5} \frac{w_{\mathrm{CD}}}{w},
$$

i. e. complete stabilization $(w \rightarrow 0)$ can be achieved in this model only with an infinite ECCD current (no effects leading to stability of small islands are considered in the above derivation). In other words, for wide CD profiles, power modulation becomes essential.

In the opposite limit $w_{\mathrm{CD}}<w_{\mathrm{marg}}$, the stabilization efficiency is approximately $\eta_{\mathrm{CD}} \lesssim$ 0.4 for both continuous injection and 50\%-duty-cycle modulation. Eq. (6) then becomes

$$
\frac{j_{\mathrm{CD}}}{j_{\mathrm{bs}}} \frac{w_{\mathrm{CD}}}{w_{\mathrm{sat}}}>\frac{1}{8} .
$$


For a saturated island width of the order of $32 \mathrm{~cm}$ (as estimated for the $q=2$ surface in ITER [11]), this condition yields

$$
\eta_{N T M} w_{\mathrm{CD}} \gtrsim 4 \mathrm{~cm}
$$

which is in quite good agreement with Sauter's stabilization criterion (2), derived in Ref. [11] and confirmed in Ref. [12] through more detailed analysis than the simple calculation presented here.

From the discussion outlined above it emerges that the two stabilization criteria $(1,2)$ correspond to different limiting situations for the relative size of the ECCD profile as compared to the marginal island width. An obvious limitation of the previous analysis is the fact that $w_{\text {marg }}$ had to be introduced $a d$ hoc in order to obtain an estimate of the CD stabilization efficiency $\eta_{\mathrm{CD}}$ in the corresponding limit. More consistently, $w_{\text {marg }}$ should appear in Eq. (3) from the beginning; moreover, the stabilization efficiency $\eta_{\mathrm{CD}}$ should be known for arbitrary values of $w / w_{\mathrm{CD}}$, as in the analysis of Refs. [11, 12]. Eq. (3) is then reformulated as

$$
0=-1+\frac{w_{\mathrm{sat}} w}{w^{2}+w_{\mathrm{marg}}^{2}}-5.05 \frac{w_{\mathrm{CD}} w_{\mathrm{sat}}}{w^{2}} \frac{j_{\mathrm{CD}}}{j_{\mathrm{bs}}} \eta_{\mathrm{CD}}\left(w / w_{\mathrm{CD}}\right),
$$

including the incomplete pressure flattening inside the island due to finite perpendicular transport [28] as a stabilizing mechanism for small islands. The results of the modelling have been shown not to depend strongly on the model adopted for small-island stability, see e. g. Ref. [12]. Eq. (10) is Eq. (12) of Sauter et al. [11], apart from the prefactors in front of the last two terms and from a different definition of the efficiency function $\eta_{\mathrm{CD}}$ :

$$
\eta_{\mathrm{CD}}\left(\frac{w}{w_{\mathrm{CD}}}\right)=\frac{w^{2}}{w_{\mathrm{CD}}^{2}} \eta_{\mathrm{CD}}^{\text {Sauter }}\left(\frac{w}{w_{\mathrm{CD}}}\right) \text {. }
$$

Using the fits reported in Ref. [30], $\eta_{\mathrm{CD}}$ can be written explicitly as

$$
\eta_{\mathrm{CD}}^{\bmod }\left(\frac{w}{w_{\mathrm{CD}}}\right)=\frac{9}{20} \tanh \left(\frac{2 w}{5 w_{\mathrm{CD}}}\right)
$$

and

$$
\eta_{\mathrm{CD}}^{\mathrm{cw}}\left(\frac{w}{w_{\mathrm{CD}}}\right)=\frac{w^{2} / w_{\mathrm{CD}}^{2}}{4\left(1+2 w^{2} / 3 w_{\mathrm{CD}}^{2}\right)}
$$

for modulated (O-point injection, 50\% duty cycle) and continuous injection, respectively. The corresponding limits,

$$
\eta_{\mathrm{CD}}^{\bmod } \longrightarrow \frac{9}{50} \frac{w}{w_{\mathrm{CD}}} \quad \eta_{\mathrm{CD}}^{\mathrm{cw}} \longrightarrow \frac{1}{4} \frac{w^{2}}{w_{\mathrm{CD}}^{2}} \quad\left(w \ll w_{\mathrm{CD}}\right)
$$

and

$$
\eta_{\mathrm{CD}}^{\bmod } \longrightarrow \frac{9}{20} \quad \eta_{\mathrm{CD}}^{\mathrm{cW}} \longrightarrow \frac{3}{8} \quad\left(w \gg w_{\mathrm{CD}}\right)
$$

for large and small deposition width agree reasonably well with the estimates used above (which are taken from Ref. [9]). 

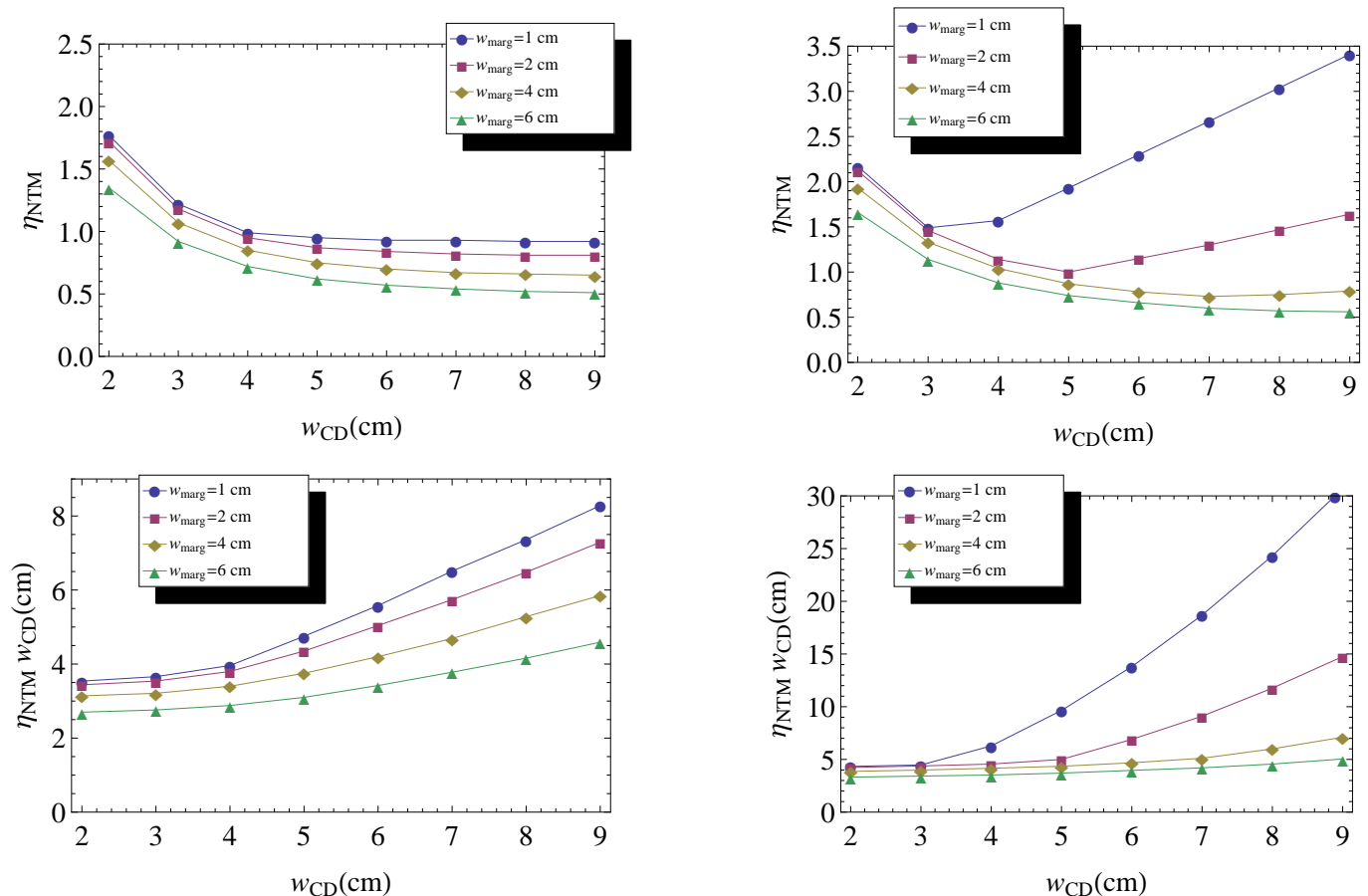

Fig. 1. The value of $\eta_{N T M}$ (upper row) and of $w_{C D} \times \eta_{N T M}$ (lower row) corresponding to unconditional stability as a function of the width of the ECCD profile for different values of $w_{\text {marg }}$ and for $w_{\text {sat }}=32 \mathrm{~cm}$. The left plots correspond to modulated ECCD, the right plots to continuous wave injection.

The value of $\eta_{N T M}$ corresponding to unconditional stability, i. e. the value at which the maximum of the right-hand side of Eq. (10) drops below zero for a given choice of $w_{\text {sat }}$ and $w_{\text {marg }}$, can be plotted for different values of $w_{\mathrm{CD}}$ to check the validity of the criteria derived above. In Fig. 1, the saturated island width is imposed to be $32 \mathrm{~cm}$ and the marginal island width is varied between 1 and $6 \mathrm{~cm}$. For large CD deposition widths the value of $\eta_{N T M}$ required for stabilization saturates to a constant level smaller than 1.2 if modulation is included, while it increases with $w_{\mathrm{CD}}$ for continuous injection. For narrow deposition profiles, on the other hand, modulation becomes unessential and $\eta_{N T M}$ scales like $1 / w_{\mathrm{CD}}$, i. e. $w_{\mathrm{CD}} \times \eta_{N T M}$ approaches a constant (in this limit, the ratio between $\eta_{N T M}^{\bmod }$ and $\eta_{N T M}^{\mathrm{cW}}$ tends to 1.2 , corresponding to the ratio $(9 / 25) /(3 / 10)$, cf. Eq. (14)).
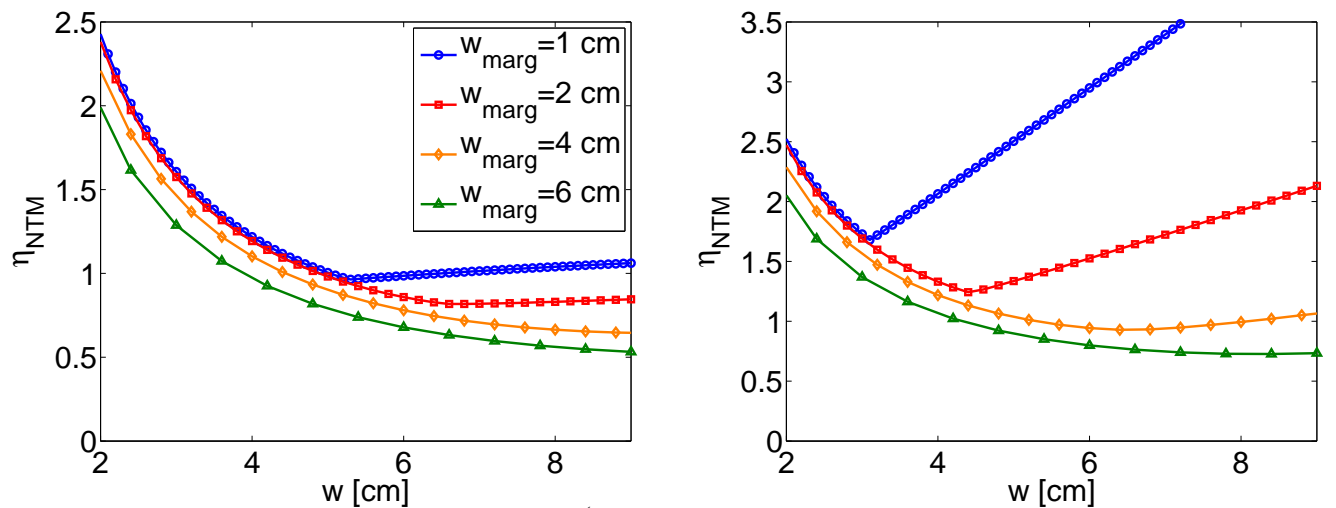

Fig. 2. The same as the first row of Fig. 1 ( $\eta_{N T M}$ corresponding to unconditional stability for modulated (left) and $\mathrm{cw}$ (right, same legend) injection), employing the equations reported in Ref. [12] (excluding the effect of heating and the modification of the linear stability parameter due to ECCD).

Similar results are obtained employing the modelling presented in Ref. [12], as shown in Fig. 2. The small differences between Fig. 1, first row, and Fig. 2 are mainly due to 
the fact that the fits of the CD stabilization efficiency used in Ref. [12] differ slightly from those of Ref. [30]. In Fig. 2, the effects due EC heating in the island region and to a change in the linear stability parameter caused by ECCD have been neglected, as they have been shown to be small.

The previous considerations confirm the validity of the stabilization criteria adopted so far, each in its range of validity. They appear to be valid also for a "worst-case" scenario in which the marginal island width is as small as 1 centimetre. In this sense they are conservative, representing rather a sufficient than a necessary condition for NTM suppression. It is finally noted that Eq. (1) and Eq. (2) lead to the same value of $\eta_{N T M}$ for NTM suppression for $w_{\mathrm{CD}}=w_{\mathrm{CD} \text {, crit }}=5 / 1.2 \simeq 4.2 \mathrm{~cm}$. For $w<w_{\mathrm{CD} \text {,crit }}$, Sauter's criterion (2) is to be employed, while Zohm's criterion (1) applies at larger $w_{\mathrm{CD}}$. Since each criterion leads to stronger requirements on $\eta_{N T M}$ (and hence on the required injected power) in its respective domain of validity, it can be inferred that $w_{\mathrm{CD}}$,crit represents a sort of optimum deposition width to be targeted in the design of the launcher. Smaller deposition widths imply "over-focusing", while deposition widths larger than $w_{\mathrm{CD}}$,crit are detrimental, as in this case some of the power is wasted outside the island during the stabilization process. Moreover, power modulation becomes increasingly important in this limit. Therefore, for an optimization of the UL it is essential to have a realistic determination of the expected deposition profiles. This point is discussed in the next section.

\section{$3 \quad$ Effects leading to broader deposition profiles}

In the past years, a number of studies have been devoted to the physical processes that could modify the EC deposition profiles as computed in ray/beam tracing modelling. The "standard" analysis is usually based on the solution of either the equations of standard geometrical optics or of extended-ray / paraxial-beam-tracing equations as far as the propagation is concerned, while absorption and current drive calculations are based on linear or quasilinear modelling [31]. In ITER, quasilinear distortions of the electron distribution function are usually found to be very small (consistently with the smallness of the Harvey parameter [32] for standard injection scenarios). Adjoint modelling of current drive including momentum-conserving corrections [33, 34], as implemented by now in several codes, also matches well Fokker-Planck results [35].

In the following, a number of beam-broadening effects are reviewed and an attempt at a quantitative assessment is performed. Processes which are predicted to have an impact on the EC profile in the range of $10 \%$ or less are discussed first, while those potentially leading to more significant broadening (of the order of 100\%) are discussed at the end of the section.

\subsection{Multi-beam effects}

As described in Sec. 1, the present design of the UL is based on the injection of four beams from each steering mirror. Due to the linearity of the problem, EC modelling is usually performed by taking a single "representative" beam for each mirror and then multiplying the result by four. In reality, it is impossible to design a system that guarantees a perfect superposition of the four beams over the whole steering range. A recent study [36] indicates that the deterioration of the real (multi-beam) deposition profile with respect to the ideal single-beam profile is in the $15 \%$ range for USM beams at the $q=3 / 2$ surface, with even better values for the LSM and the $q=2$ surface, and with margin for further optimization.

\subsection{Quasi-linear effects in the presence of an island}

The deposition profiles are usually computed assuming an axisymmetric geometry with unperturbed flux surfaces. However, in the presence of a magnetic island, new flux surfaces 
are formed and when the EC heating aims at its O-point, the power density could be such that quasilinear effects become relevant. Recent investigations show that indeed the Harvey parameter can exceed the threshold for quasilinear effects in a vicinity of the Opoint. The subsequent quasilinear flattening reduces the absorption rates and shifts the absorption profile to regions of smaller CD efficiency, reducing the total driven current [37]. This effect has been found to be relatively small in present-day machines and should be negligible in ITER, where the increase in plasma volume will largely overcompensate the increased EC power.

\subsection{Aberration}

A new formalism that aims to account for aberration effects, i. e. modifications of wave propagation with respect to a paraxial description, has been developed recently [13]. Application of a new quasi-optical code implementing this formalism to ITER-like scenarios quantified the possible broadening of the EC profiles due to aberration in the range between 10-20\% [14]. The physical origin of the broadening has been identified in a combination of spatial dispersion and strong absorption [15]: due to the variation of $k_{\|}$(component of the wave vector parallel to the magnetic field) across the beam cross section, part of the beam can be absorbed earlier than in the paraxial limit, where only the value of $k_{\|}$ at the centre of the beam is taken into account. A simplified model aiming at mimicking the ITER injection geometry, developed in Ref. [15], has confirmed that beam broadening due to aberration is in the range of $\lesssim 10 \%$. As a side note, it is remarked that asymmetric absorption of the beam with respect to its axis, arising naturally for oblique incidence onto the resonance as in the case of the ITER UL, is found not to lead to any noticeable distortion of the beam trajectory with respect to standard beam tracing calculations [38].

\subsection{Radial transport of fast electrons}

In hot plasmas, the collisional time scale on which the current drive profile arising from the Fisch-Boozer effect [39] establishes can be comparable with the time on which the heated electrons diffuse away from the region where the power is absorbed from the wave beam. This effect has been shown to be able to explain experimentally observed profiles in L-mode TCV discharges [40], for diffusion coefficients of $3.7 \mathrm{~m}^{2} / \mathrm{s}$. An analysis performed for ITER parameters indicates that diffusion coefficients of the order of $1 \mathrm{~m}^{2} / \mathrm{s}$ would broaden the CD profile determined from ray/beam tracing calculations by a factor of two [16], implying a reduction of the peak $j_{\mathrm{CD}}$ of the driven current density by the same factor. In Ref. [16], the diffusion coefficient $D$ of the ECCD electrons was treated as a free parameter and an analytic formula was proposed to determine the reduction of $j_{\mathrm{CD}}$ in the presence of transport (Eq. (10) of Ref. [16]):

$$
\frac{j_{\mathrm{CD}}}{j_{\mathrm{CD}, 0}}=\frac{w_{\mathrm{CD}}}{\sqrt{w_{\mathrm{CD}}^{2}+4 D \tau}}
$$

where $j_{\mathrm{CD}, 0}$ and $w_{\mathrm{CD}}$ are the peak value of the driven current density and the full width of the CD profile in the absence of transport, respectively, and $\tau$ is the collision time of the current-carrying electrons. This formula makes explicit the obvious fact that the relative reduction of the peak current is strongest (for a given diffusion coefficient $D$ ) when the deposition profile in the absence of transport is narrowest. The value of $j_{\mathrm{CD}} / j_{\mathrm{CD}, 0}$ from Eq. (15) is plotted against $w_{\mathrm{CD}}$ in Fig. 3 for different values of the diffusion coefficient $D$. For $w_{\mathrm{CD}}$ in the range of $3-4 \mathrm{~cm}$, as predicted by beam tracing codes for typical ITER parameters, see Sec. 4, the reduction of the peak current density by a factor of two for $D=1 \mathrm{~m}^{2} / \mathrm{s}$ can be observed. If, on the other hand, other physical effects lead to wider beams in the region of resonant absorption (like the beam scattering from density fluctuations described below), the impact of finite transport is reduced. 


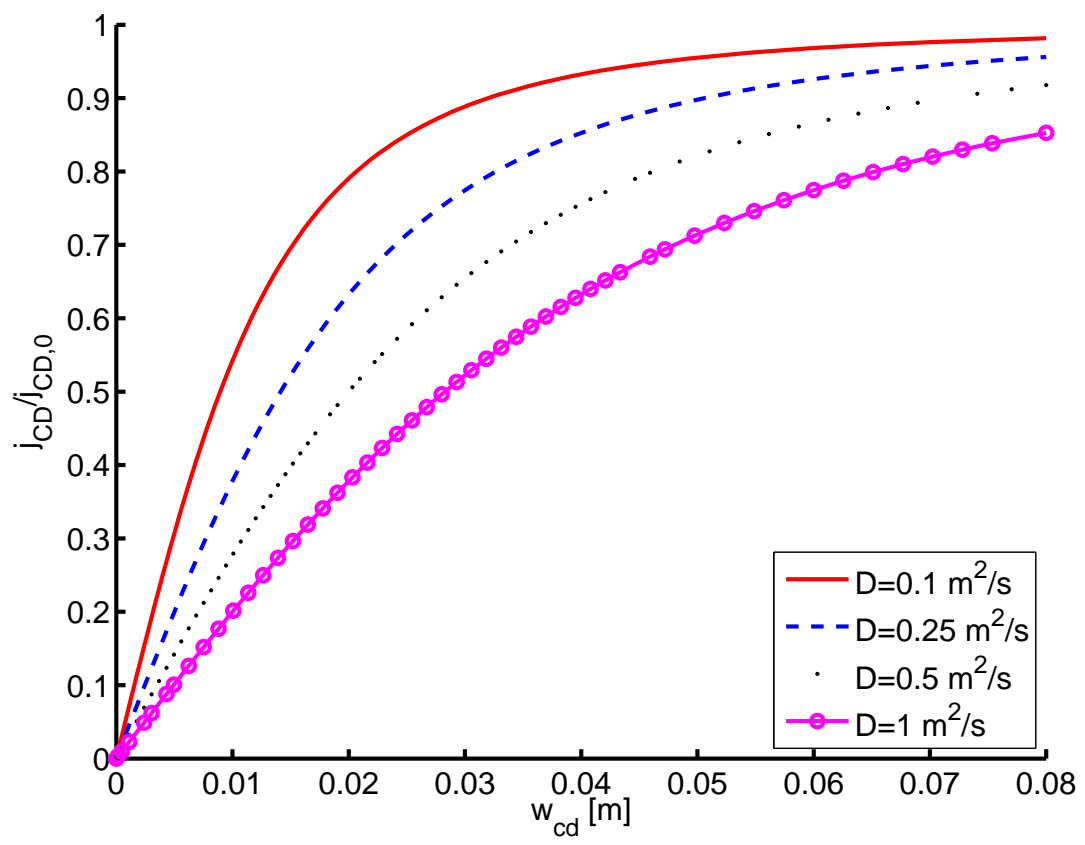

Fig. 3. Blackuction of the $j_{C D}$ peak due to radial transport as a function of the full deposition width according to Eq. (15) for $\tau=6 \cdot 10^{-4} \mathrm{~s}$ (typical of electrons at 2.5 times the thermal velocity for ITER parameters at the $q=2$ surface).

Very recently, the direct numerical simulation of the turbulent transport for parameters typical of the $q=3 / 2$ and $q=2$ surfaces in ITER has been addressed [41] employing the gyrokinetic code GKW [42], with the goal of determining some constraints on the range of possible values attained by the diffusion coefficient $D$ in the region of velocity space affected by the interaction with the EC beam. The values of $D$ found in the simulations for passing electrons with a velocity between 1.5 and 2.5 times the thermal velocity are in the range of $0.1-0.2 \mathrm{~m}^{2} / \mathrm{s}$. These GKW simulations include electromagnetic effects on the small-scale fluctuations, but neglect the presence of the magnetic island. This implies that the estimates given above are to be understood as an upper limit on $D$, since inside an island the kinetic profiles are flattened and the drive for the turbulence is strongly reduced $[43,44]$ (although this might be only partly the case for islands of about the marginal island size). These low values of the diffusion coefficient, in combination with the beam broadening discussed in the next subsection, suggest a relatively small role (again at the 10\%-level) of transport effects on the determination of the CD profiles for the UL in ITER.

\subsection{Wave scattering from density fluctuations}

It was recognized long ago (see e. g. Ref. [45]) that small deviations of the beam path due to density fluctuations can have a sizeable effect in large fusion devices, where the waves can propagate over a large distance after having been deflected. The modelling of this effect is very challenging for the theory, since in general small-scale fluctuations break down the WKB ordering on which usual ray/beam tracing calculations rely, and a direct solution of the integro-differential wave equation including absorption, on the computational domain required for heating and current drive scenarios in large-size tokamak applications, is still not affordable. Recent attempts at quantifying scattering-induced beam broadening, based on a Fokker-Planck modelling of the diffusion of rays $[17,46]$ or on ray tracing in the presence of fluctuations that satisfy WKB ordering [18] suggest an increase of the beam width at the absorption layer in ITER applications of the order of $100 \%$. The scattering process has also been addressed through a solution of the the full wave equations in the 
presence of simplified blob geometry [47].

In an even more recent work, the problem has been addressed employing a more sophisticated approach, based on the solution of the wave kinetic equation in the presence of small-scale fluctuations [48], retaining diffraction, full tokamak geometry and determination of the power absorption profile. More specifically, the steady-state wave kinetic equation is solved,

$$
\{H, W\}=-2 \gamma W+S(\Gamma, W),
$$

where, in the Poisson brackets on the left-hand side, $H$ is the standard geometric-optics Hamiltonian for the mode under consideration, $W$ is the Wigner function, which can be interpreted as a measure of the wave-field energy density ( $W$ is the Weyl symbol of the electric field correlation in the semiclassical limit). Furthermore, $\gamma$ is the absorption coefficient and $S$ the scattering operator, which depends on the Wigner transform $\Gamma$ of the density correlation (see Refs. $[49,50]$ for details). As in previous studies $[17,18]$, the turbulence is modelled as a layer (of width $\Delta \rho_{F}$ ) of density fluctuations with $\delta n_{e} / n_{e}$ in the 10\%-range located at the plasma periphery. The first results obtained by means of a numerical solution of Eq. (16) obtained by means of the new code WKBeam [51] indicate a broadening of the ECCD profile by a factor of two or even larger, depending on the parameters chosen in the simulations, see Fig. 4. This broadening is in the same range as previous estimates. Hence, although a final statement on the subject is premature, see also discussion below, it is argued that this effect is likely to lead to the most severe loss of localization of the EC deposition profiles for NTM stabilization in ITER.
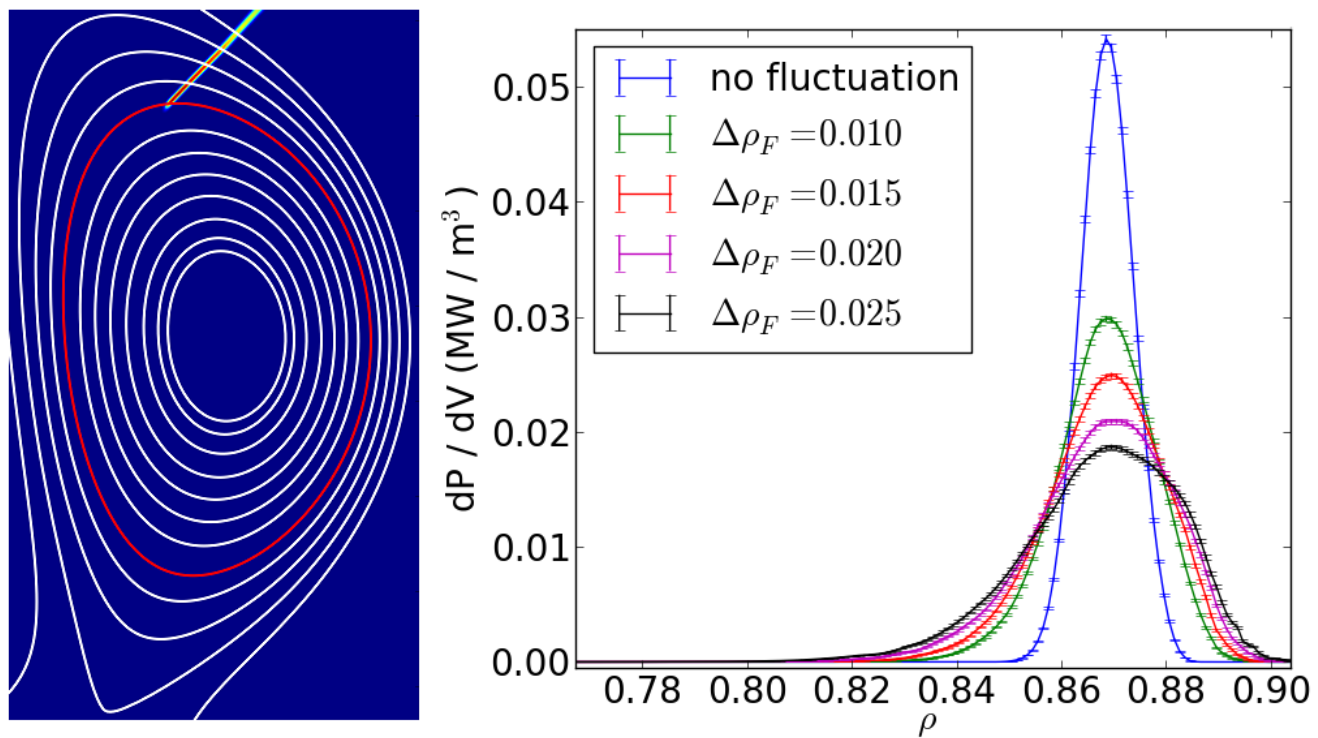

Fig. 4. Poloidal projection of the propagation path of an EC beam for NTM stabilization in ITER (left) and broadening of the power deposition profile in ITER due to density fluctuations for different widths of a turbulent layer centred around the separatrix (right). The fluctuation level is $\delta n_{e} / n_{e}=0.1$ and the turbulence correlation length perpendicular to the equilibrium magnetic field is $L_{\perp}=2 \mathrm{~cm}$. Results of the wave kinetic equation solver WKBeam.

Unfortunately, in present-day machines, this effect is predicted to be much smaller, first of all because of their smaller size, but also because of the differences in the transport regime $[49,50]$. This makes an experimental confirmation of the theoretical calculations very challenging. Moreover, the modelling based on the wave-kinetic equation sketched above also contains approximations (particularly in the treatment of the scattering term and the related Born approximation $[52,48]$ ) whose validity still requires a careful assessment. More detailed studies will be needed to achieve better quantitative predictions. 


\section{Impact of profile broadening and misalignment on the power requirements for NTM control in ITER}

The impact of current-profile broadening on the power requirements for NTM suppression are now estimated by a direct solution of Eq. (10). From the discussion of Sec. 2, it is clear that an increase of the $\mathrm{CD}$ deposition profile width at constant total driven current does not imply necessarily an increase of the power needed for complete stabilization of the mode. As far as the driven current remains well confined inside the island, the impact of profile broadening remains small. Whether this will be the case during the stabilization process, depends mainly on the marginal island size, whose value in extrapolations to ITER is still affected by a large uncertainty.

With respect to Eq. (10), in the helical efficiency $\eta_{C D}$ we now include also the effect of a possible misalignment between the resonant surface on which the mode develops and the position at which the maximum current density is driven. This is done by assuming a current profile of the form (see Ref. [30])

$$
J_{\mathrm{CD}}=j_{\mathrm{CD}} \exp \left[-\frac{4 x_{\mathrm{mis}}^{2}}{w_{\mathrm{CD}}^{2}}\right]
$$

(where $x_{\text {mis }}=\left|\rho-\rho_{\mathrm{CD}}\right|$ ) and inserting it in Eq. (4). The integrals in Eq. (4) have to be performed numerically and the result is shown in Fig. 5 for continuous wave (cw) and modulated O-point injection with $50 \%$ duty cycle. It is noted that the numerical results for $50 \%$ duty cycle at zero misalignment saturate at a slightly lower value than the fit used in Sec. 2.

$\eta_{\mathrm{cd}}, \mathrm{CW}$ injection

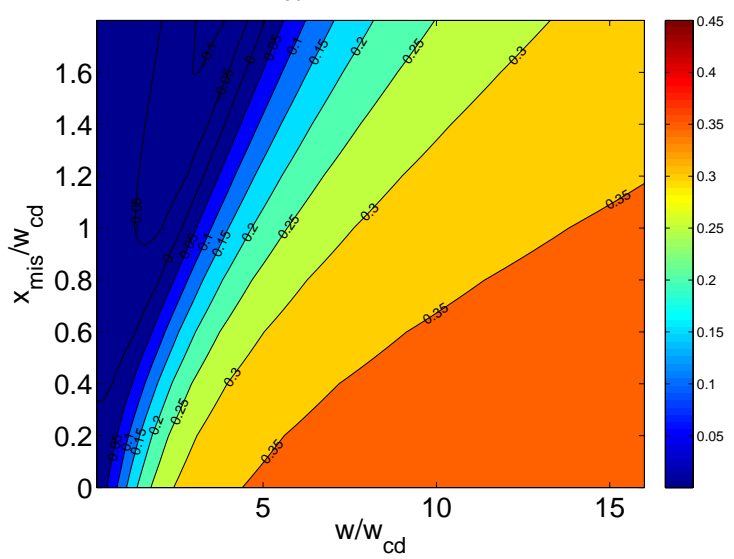

$\eta_{\text {cd }}, 50 \%$ duty cycle

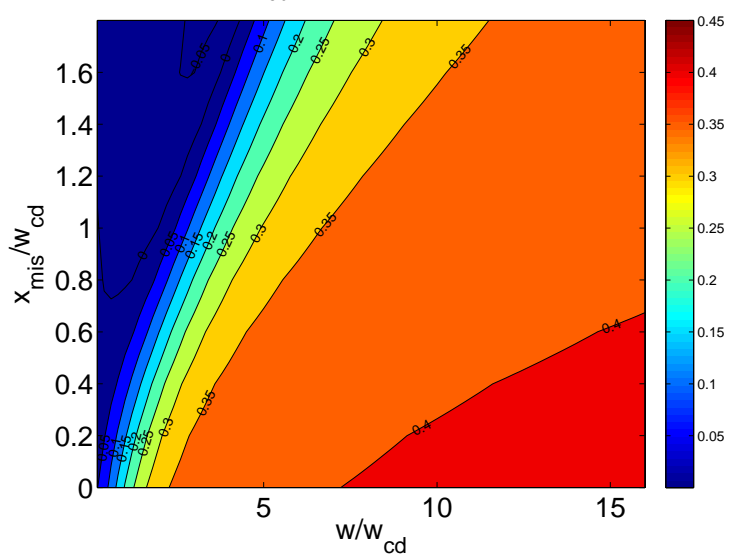

Fig. 5. The helical NTM stabilization efficiency defined in Eq. (4) as a function of the island width and the profile misalignment normalized to the deposition width for continuous wave (left) and modulated injection (right). In the case of modulation, $\eta_{C D}$ has been divided by two to account for the reduced time the current is turned on (50\% duty cycle considered).

It is worth stressing that for islands of the size of the CD profile width $w_{\mathrm{CD}}$, a misalignment in the range $0.6-0.8 \times w_{\mathrm{CD}}$ (depending on whether modulated or $\mathrm{cw}$ injection is considered) leads to a drop of the helical efficiency below zero, implying a loss of the stabilizing effect of ECCD. It is therefore mandatory that the steering system has a sufficient accuracy to compensate for misalignments in this range $[53,54,55]$. The steering system for the ITER UL is designed to ensure a steering accuracy $\Delta \alpha=0.1^{\circ}$. According to beam tracing calculations, this corresponds to a shift of the deposition profile in the range $\Delta \rho=0.001-0.002$ in normalized flux coordinate (the square root of the toroidal flux is usually employed in our analysis), i. e. $2-4 \mathrm{~mm}$ if measured in the outer midplane. This corresponds to $0.1 \times w_{\mathrm{CD}, 0}$ at most, where $w_{\mathrm{CD}, 0}$ is the width obtained from beam tracing calculations in the absence of profile-broadening effects (cf. Table 1 below). 
This means that even in the case of no broadening, the steering mechanism should ensure sufficient compensation for possible misalignments (a more detailed analysis of this issue will be presented elsewhere [56]). The real challenge for the ITER control system (for scenarios in which ECCD is applied after NTM detection and not pre-emptively) will be rather to determine $[57,58]$ and reach the correct location in a sufficiently short time, so as to avoid mode locking $[53,55]$.
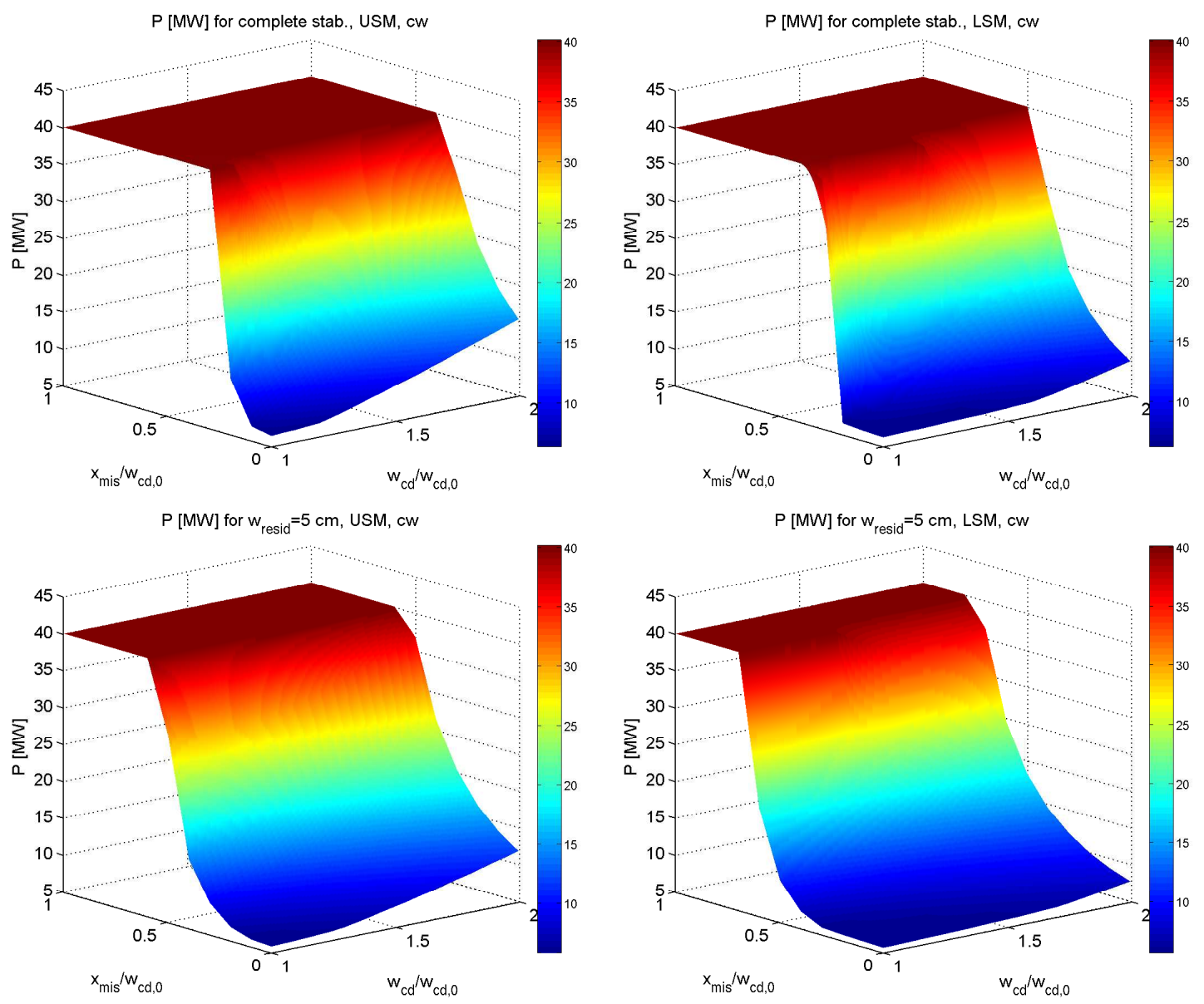

Fig. 6. Power requirements for complete NTM stabilization (upper row) or reduction of the island width to $5 \mathrm{~cm}$ (lower row) as a function of the profile broadening and of the misalignment for the $q=2$ surface. The left column refers to the USM, the right one to the LSM. CW injection is considered, with $w$ marg $=2 \mathrm{~cm}$.

The plasma parameters considered here have been taken from a snapshot (close to the end of the current flattop phase) of a simulated plasma discharge for the standard $I_{p}=15$ MA, $Q=10$ ITER scenario [59], while the ECCD parameters have been computed with the code TORBEAM [60] (a detailed analysis of the ECCD performance for this and other simulated ITER discharges will be presented in Ref. [56]). The values of $w_{\text {sat }}$ are taken from Ref. [11], as mentioned above, while $w_{\text {marg }}$ is varied between 1 and $6 \mathrm{~cm}$ (in figures where the scans for a fixed value of $w_{\text {marg }}$ are presented, the value $w_{\text {marg }}=2 \mathrm{~cm}$ has been chosen). The values employed for the solution of the Rutherford equation (10) are reported in Table 1.

\begin{tabular}{|c|c|c|c|c|c|c|c|}
\hline$q$ & $n_{e} / 10^{19}$ & $T_{e}$ & $\eta_{N T M, U S M}$ & $\eta_{N T M, L S M}$ & $w_{\mathrm{CD}, \mathrm{USM}, 0}$ & $w_{\mathrm{CD}, \mathrm{LSM}, 0}$ & $w_{\text {sat }}$ \\
\hline $3 / 2$ & $9.72 \mathrm{~m}^{-3}$ & $8.77 \mathrm{keV}$ & 0.21 & 0.24 & $4.7 \mathrm{~cm}$ & $4.0 \mathrm{~cm}$ & $25 \mathrm{~cm}$ \\
\hline 2 & $9.56 \mathrm{~m}^{-3}$ & $7.06 \mathrm{keV}$ & 0.21 & 0.27 & $3.7 \mathrm{~cm}$ & $2.8 \mathrm{~cm}$ & $32 \mathrm{~cm}$ \\
\hline
\end{tabular}

Table 1: Parameters employed in the numerical solution of the Rutherford equation (10). The values of $\eta_{N T M}=j_{C D} / j_{b s}$ are per $M W$ of absorbed power. The deposition widths are obtained by 
multiplying the widths in units of $\rho$ (square root of the normalized toroidal flux) by the midplane minor radius $a=201 \mathrm{~cm}$.

The power requirement for NTM stabilization according to a numerical solution of Eq. (10) is shown in Fig. 6 for $q=2$ parameters for $\mathrm{cw}$ injection and $w_{\text {marg }}=2 \mathrm{~cm}$. In the upper row, the power needed for complete suppression has been calculated while the second row shows the power needed to reduce the island size below the threshold $(5 \mathrm{~cm}$ for $q=2,8 \mathrm{~cm}$ for $q=3 / 2$ ) predicted for mode locking in Ref. [53]. The misalignment and the CD profile width are normalized to the nominal profile width $w_{\mathrm{CD}, 0}$. The calculation is stopped when the required power exceeds $40 \mathrm{MW}$. In the case of no misalignment, it can be seen that the power needed for complete NTM stabilization is not strongly affected by a broadening of the deposition profile, as long as it satisfies $w_{\mathrm{CD}} \lesssim w_{\mathrm{CD}}$,crit, where $w_{\mathrm{CD} \text {, crit }}$ has been defined at the end of Sec. 2 . These are the regions $w_{\mathrm{CD}} \lesssim 1.15 w_{\mathrm{CD}, 0}$ for the USM and $w_{\mathrm{CD}} \lesssim 1.5 w_{\mathrm{CD}, 0}$ for the LSM (the LSM delivers more focused beams in the region of the plasma prone to NTMs and leads hence to narrower deposition profiles). As mentioned previously, this behaviour is due to the fact that for deposition below $w_{\mathrm{CD}}$,crit the stabilization is determined by the total driven current, which is not affected by a simultaneous broadening of the profile and reduction of its peak value, assuming that the current drive efficiency does not change significantly on the scale of the beam width. For deposition widths larger than $w_{\mathrm{CD}}$,crit, the power requirements increase, since now a considerable fraction of the current is driven outside the island. In the case of the USM, a doubling of the nominal deposition profile (with the related decrease of $j_{\mathrm{CD}}$ by the same amount) brings the power requirement in the range of the maximum available power from a given row of mirrors (13.3 MW, see Sec. 1).


Fig. 7. The same as Fig. 6 for modulated injection. 
The power requirements are decreased if just a reduction of the island size, rather than full suppression, is targeted. Moreover, in this case also the misalignment poses less severe constraints on the steering accuracy. In the case of modulated injection, the broadening of the current profile leads to a much smaller increase of the stabilization power with the profile width, as shown in Fig. 7. Also the power increase with misalignment is less steep as in the $\mathrm{cw}$ case. The situation is summarized in Fig. 8, where the power required for NTM stabilization at the $q=2$ surface (for zero misalignment) is plotted for different values of $w_{\text {marg }}$, assuming nominal CD profiles (left) and a doubling of the profile width (right).
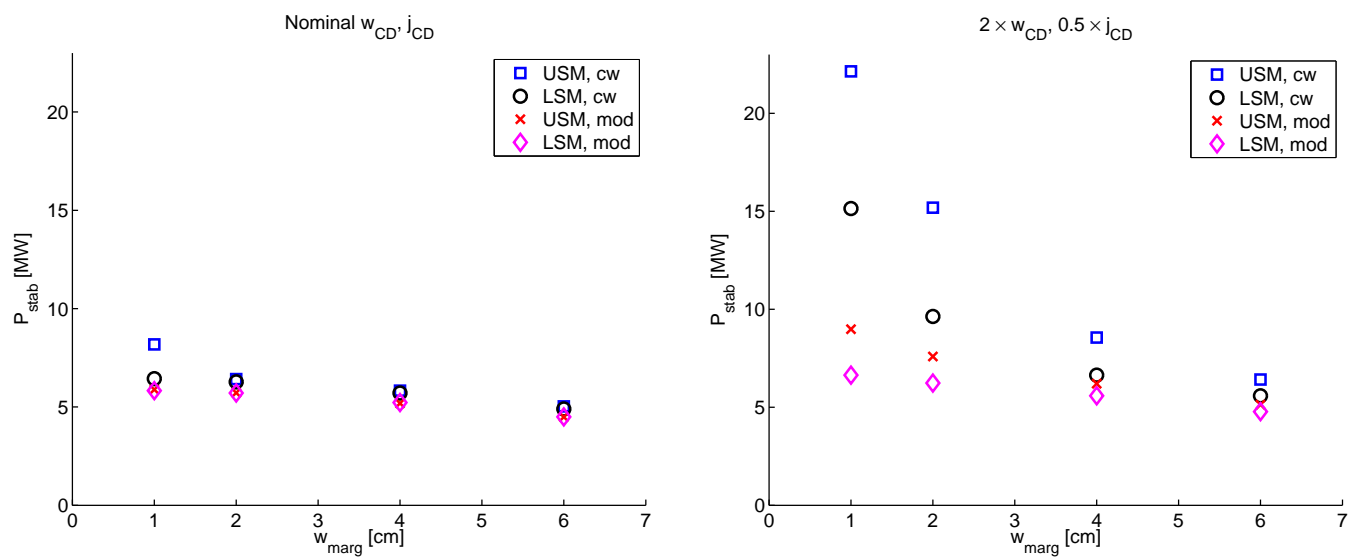

Fig. 8. Power required for complete NTM suppression on the $q=2$ surface for different values of the marginal island size $w_{\text {marg }}$ for the case of nominal CD profiles (left) and broadening of the profile by a factor two (right).

For the scenario and time slice under consideration, it can be seen that the stabilization power is well within the $13.3 \mathrm{MW}$ allocated for each row of mirrors if $w_{\text {marg }} \gtrsim 4 \mathrm{~cm}$. For lower values of $w_{\text {marg, }}$, the required power can exceed this threshold if $\mathrm{cw}$ injection is applied and the peak driven current density $j_{\mathrm{CD}}$ is assumed to decrease by a factor of two with respect to its nominal value. Very similar conclusions can be drawn for the case of the $q=3 / 2$ surface (the corresponding plasma parameters are shown for completeness in Table 1. More detailed results are reported in Ref. [61]).
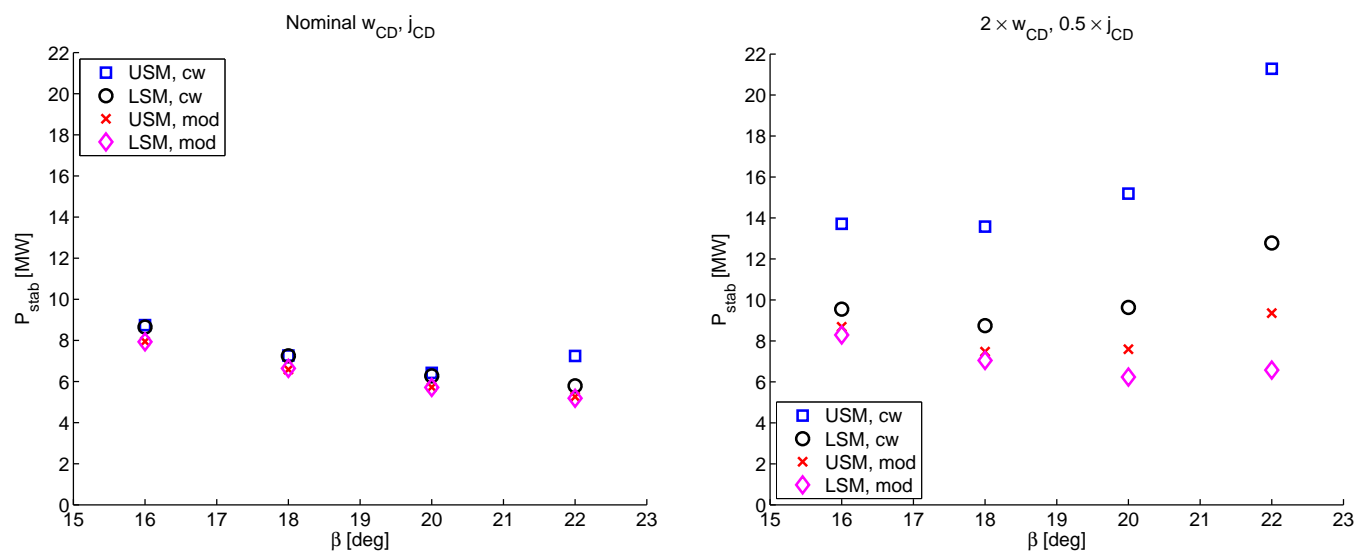

Fig. 9. Impact of the toroidal launch angle $\beta$ on the power requirements for NTM suppression for nominal beam tracing values (left) and for profile broadening by a factor of two (right). Plasma and beam parameters for the $q=2$ surface and $w \operatorname{marg}=2 \mathrm{~cm}$.

Finally, the impact of profile broadening on the optimum toroidal injection angle is displayed in Fig. 9 for $w_{\operatorname{marg}}=2 \mathrm{~cm}$ and $q=2$. The toroidal angle $\beta$ has been varied between $16^{\circ}$ and $22^{\circ}$ and the power for NTM suppression has been evaluated from nominal 
beam tracing profiles and assuming (somehow arbitrarily) for each of them a broadening by a factor of two. It can be observed that for nominal profiles the optimum value of $\beta$ (corresponding to minimum power requirements) is in the range $\beta \gtrsim 20^{\circ}$. The minimum value of the stabilization power as a function of $\beta$ arises from a balance between narrowness of the ECCD profile (decreasing with $\beta$ ) and total driven current (increasing with $\beta$ ), as explained above when discussing the optimum profile width $w_{\mathrm{CD} \text {, crit }}$. In particular, for the LSM an increase of $\beta$ would appear desirable, as suggested in Ref. [12]. If profile broadening is assumed, however, our calculations show a strong increase of the stabilization power for $\beta>20^{\circ}$ and the optimum $\beta$ is found rather around $18^{\circ}$ for $\mathrm{cw}$ injection. Due to the uncertainties on the size of the profile broadening, it appears advisable at this stage not to change the present design value $\beta=20^{\circ}$.

\section{Discussion and conclusions}

The results presented in this paper allow us to draw some important conclusions. First of all, the validity of the criteria for NTM stabilization in ITER, employed so far as a guidance for the design of the EC upper launcher, has been confirmed. Their physical content (stating that the total driven current is the quantity to be maximized if the deposition profile is well inside the magnetic island, the driven current density is to be maximized in the opposite case) can be derived straightforwardly from the terms in the Rutherford equation that according to our present understanding are the most relevant for the determination of NTM stability in ITER, i. e. those involving the stability parameter $\Delta^{\prime}$, the neoclassical bootstrap drive and the stabilization through the helical component of the EC driven current. From a quantitative point of view, these criteria are expected to represent sufficient conditions for NTM suppression, but are not meant to replace a more detailed NTM simulations. In this respect, however, our predictive capabilities are still impaired by various factors, related to the modelling of EC waves on one hand and to that of NTMs on the other hand. Concerning the first aspect, it has been argued in this paper that the main factor inhibiting the extremely good localization of the EC power deposition predicted by standard beam tracing analysis for the UL beams will be the scattering of the waves from density fluctuations due to plasma turbulence. Despite the fact that a final quantitative assessment of this effect is still not yet possible, there has been a constant refinement of the physical and numerical modelling, leading to a growing evidence that a significant beam broadening (by a factor of two or possibly more) is to be expected under ITER conditions. Concerning the physics of NTMs, as our present uncertainties concentrate on the stability of small islands, the marginal island width has been treated as a free parameter, and only the reduction of the bootstrap drive due to finite perpendicular transport has been retained. A simplified form of the Rutherford equation, including the terms mentioned above without fine-tuning of the respective coefficients, has been used to determine the power requirements for NTM stabilization for a representative set of ITER parameters, corresponding to the end of the flattop phase of the standard ELMy H-mode scenario. The CD profile width has been varied between one and two times its nominal value (given by standard beam tracing predictions) and $w_{\text {marg }}$ between 1 and $6 \mathrm{~cm}$. Moreover, the misalignment between the peak of the deposition profile and the resonant flux surface has been taken into account.

The results of the modelling indicate that the power available for NTM stabilization should be largely sufficient for suppression of NTMs in the flattop phase of a standard ITER ELMy H-mode scenario, when the current density profiles from standard beam tracing calculations are considered. The validity of this statement becomes marginal (for $\mathrm{cw}$ injection), if $w_{\mathrm{marg}}$ is of the order of 1-2 $\mathrm{cm}$ and for a broadening of the CD profile by a factor of two (with the consequent reduction of $j_{\mathrm{CD}}$ by the same factor). A possible implication of this result is that power modulation for NTM suppression could become necessary. In this case, one should take into account that the time needed for the generation of ECCD through the Fisch-Boozer mechanism could become of the same time as the 
island rotation time in front of the launcher. Due to the significant degradation of the stabilization efficiency in the case of large CD profile broadening, it seems recommendable not to increase the toroidal injection angle from the value foreseen by the present design. Finally, the steering accuracy envisaged for the EC UL should be sufficient to compensate for possible misalignment, provided that the plasma control system is able to detect the mode, determine its location and react on a sufficiently fast time scale.

A more complete analysis of entire simulated ITER discharges for different scenarios should be performed to assess the degree of generality of these conclusions.

\section{Acknowledgments}

E. Westerhof is warmly acknowledged for useful discussions and for making available to us the routines used to produce the results presented in Ref. [12] and reported in Fig. 2. We wish also to thank C. Rapson for useful comments and suggestions.

This work has been performed within Fusion for Energy Grant 161. The views expressed in this publication are the sole responsibility of the author and do not necessarily reflect the views of Fusion for Energy.

\section{References}

[1] OMORI, K. et al., Fusion Engineering and Design 86 (2011) 951.

[2] HENDERSON, M. A. et al., Fusion Engineering and Design 82 (2007) 454.

[3] STRAUSS, D. et al., Fusion Engineering and Design 88 (2013) 2761.

[4] GRASWINCKEL, M. F. et al., Nuclear Fusion 48 (2008) 054015.

[5] ZOHM, H. et al., Journal of Physics: Conference Series 25 (2005) 234.

[6] RAMPONI, G. et al., Nuclear Fusion 48 (2008) 054012.

[7] RUTHERFORD, P. H., Physics of Fluids 16 (1973) 1903.

[8] SAUTER, O. et al., Physics of Plasmas 4 (1997) 1654.

[9] ZOHM, H. et al., Plasma Physics and Controlled Fusion 49 (2007) B341.

[10] GÜNTER, S. et al., Nuclear Fusion 38 (1998) 1431.

[11] SAUTER, O. et al., Plasma Physics and Controlled Fusion 52 (2010) 025002.

[12] BERTELLI, N. et al., Nuclear Fusion 51 (2011) 103007.

[13] BALAKIN, A. A. et al., Journal of Physics D: Applied Physics 40 (2007) 4285.

[14] BERTELLI, N. et al., Nuclear Fusion 50 (2010) 115008.

[15] MAJ, O. et al., Plasma Physics and Controlled Fusion 52 (2010) 085006.

[16] BERTELLI, N. et al., Nuclear Fusion 49 (2009) 095018.

[17] TSIRONIS, C. et al., Physics of Plasmas 16 (2009) 112510.

[18] PEYSSON, Y. et al., Plasma Physics and Controlled Fusion 53 (2011) 124028.

[19] GIRUZZI, G. et al., Nuclear Fusion 39 (1999) 107.

[20] AYTEn, D. et al., Physics of Plasmas 19 (2012) 092506.

[21] STRAUSS, D. et al., Fusion Engineering and Design 89 (2014) 1669.

[22] FURTH, H. P. et al., Physics of Fluids 6 (1963) 459.

[23] QU, W. X. et al., University of Wisconsin Plasma Report UWPR 85-5 (1985). 
[24] CARRERA, R. et al., Physics of Fluids 29 (1986) 899.

[25] GLASSER, A. H. et al., Physics of Fluids 18 (1975) 875.

[26] KOtschenreuther, M. et al., Physics of Fluids 28 (1985) 294.

[27] HEGNA, C. C. et al., Physics of Plasmas 4 (1997) 2940.

[28] FITZPATRICK, R., Physics of Plasmas 2 (1995) 825.

[29] WILSON, H. R. et al., Physics of Plasmas 3 (1996) 248.

[30] SAUTeR, O., Physics of Plasmas 11 (2004) 4808.

[31] PRATER, R. et al., Nuclear Fusion 48 (2008) 035006.

[32] HARVEY, R. W. et al., Physical Review Letters 62 (1989) 426.

[33] MARUShCHENKO, N. B. et al., Nuclear Fusion 48 (2008) 054002.

[34] MARUShCHENKO, N. B. et al., Nuclear Fusion 49 (2009) 129801.

[35] MARushchenko, N. B. et al., Physics of Plasmas 18 (2011) 032501.

[36] MORO, A. et al., AIP Proc. 1580 (2014) 550.

[37] AYTEN, D. et al., Nuclear Fusion 54 (2014) 073001.

[38] MAJ, O. et al., Physics of Plasmas 16 (2009) 062105.

[39] FISCH, N. J. et al., Physical Review Letters 45 (1980) 720.

[40] HARVey, R. W. et al., Physical Review Letters 88 (2002) 205001.

[41] CASSON, F. J. et al., Nuclear Fusion 55 (2015) 012002.

[42] PEeters, A. G. et al., Computer Physics Communications 180 (2009) 2650.

[43] POLI, E. et al., Nuclear Fusion 49 (2009) 075010.

[44] HORnsby, W. A. et al., Physics of Plasmas 17 (2010) 092301.

[45] HANSEN, F. R. et al., Nuclear Fusion 28 (1988) 769.

[46] HIZANIDIS, K. et al., Physics of Plasmas 17 (2010) 022505.

[47] RAM, A. K. et al., Physics of Plasmas 20 (2013) 056110.

[48] MCDONALD, S. W., Physical Review A 32 (1985) 1708.

[49] WEBER, H., IPP Report 5/134, 2013.

[50] WEBER, H. et al., Proceedings of the 18th Workshop on ECE and ECRH, Nara (Japan), April 22-25 (2014) to appear in EPJ Web of Conferences.

[51] WEBER, H. et al., Computer Physics Communications (2014) in preparation.

[52] KARAL, F. C. et al., Journal of Mathematical Physics 5 (1964) 537.

[53] LAHAYE, R. J. et al., Nuclear Fusion 48 (2008) 054004.

[54] URSO, L. et al., Nuclear Fusion 50 (2010) 025010.

[55] VAN DEN BRAND, H. et al., Plasma Physics and Controlled Fusion 54 (2012) 094003.

[56] FIGINI, L. et al., Plasma Physics and Controlled Fusion (2015) in preparation.

[57] VAN DEN BRAND, H. et al., EPJ Web of Conferences 32 (2012) 03004.

[58] SNIPES, J. A. et al., Fusion Engineering and Design 87 (2012) 1900.

[59] PARAIL, V. et al., Nuclear Fusion 53 (2013) 113002.

[60] POLI, E. et al., Computer Physics Communications 136 (2001) 90.

[61] POLI, E. et al., Proceedings of the 18th Workshop on ECE and ECRH, Nara (Japan), April 22-25 (2014) to appear in EPJ Web of Conferences. 